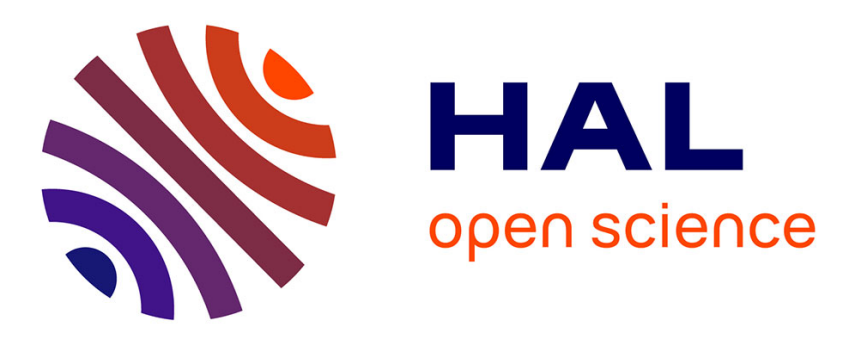

\title{
Exhaustive Modal Analysis of Large-Scale Interconnected Power Systems with High Power Electronics Penetration
}

Mohamed Kouki, Bogdan Marinescu, Florent Xavier

\section{- To cite this version:}

Mohamed Kouki, Bogdan Marinescu, Florent Xavier. Exhaustive Modal Analysis of Large-Scale Interconnected Power Systems with High Power Electronics Penetration. IEEE Transactions on Power Systems, In press, 10.1109/TPWRS.2020.2969641 . hal-02510520

\author{
HAL Id: hal-02510520 \\ https://hal.science/hal-02510520
}

Submitted on 18 Mar 2020

HAL is a multi-disciplinary open access archive for the deposit and dissemination of scientific research documents, whether they are published or not. The documents may come from teaching and research institutions in France or abroad, or from public or private research centers.
L'archive ouverte pluridisciplinaire HAL, est destinée au dépôt et à la diffusion de documents scientifiques de niveau recherche, publiés ou non, émanant des établissements d'enseignement et de recherche français ou étrangers, des laboratoires publics ou privés. 


\title{
Exhaustive Modal Analysis of Large-Scale Interconnected Power Systems with High Power Electronics Penetration
}

\author{
Mohamed Kouki, Bogdan Marinescu, and Florent Xavier
}

\begin{abstract}
Eigencalculation is a challenging task in large-scale power systems with high power electronics penetration for at least two reasons. First, the well-known inter-area modes are no longer the only coupling modes as such couplings may involve also power converters. Next, it is difficult to find all coupling modes without a priori knowledge about them (frequency or path of oscillation). In this paper we propose a new method to overcome these difficulties. It is fully analytic, i.e., does not need operator manipulations like dynamic simulations, and it is exhaustive in the sense that makes a full scan of the system for coupling modes. The approach involves concepts from matrix computation and dynamic systems analysis which hold in largescale and need no hypothesis (like the one about large inertia generators usually associated to inter-area modes) or knowledge about the structure of the power system. Validations on several models are presented, including realistic large-scale model (more than 1000 generators/dynamic devices) of the European power system.
\end{abstract}

Index Terms-Coupling/Inter-Area Modes, Modal Analysis, Eigencalculation, Large-Scale Power Systems, Power Electronics.

\section{INTRODUCTION}

Modern power systems are evolving due, in particular, to new grid interconnections, Power Park Modules (PPM), i.e., sources connected to the grid (Renewable Energy (RE), Distributed Generation (DG), storage, etc) by power electronics and FACTS (STATCOM, HVDC,...). They have thus several types of dynamic devices, i.e., components of the system with models which consist of differential equations. In the past, this class was quasi exclusively composed by classic synchronous generators.

This has a strong impact on modal analysis, i.e., computation of eigenvalues and eigenvectors in order to determine the main oscillatory dynamics of the systems and the parts (variables) involved in.

First, most of the aforementioned grid components consist of power electronic devices (power converters) or are connected to the grid via such elements. This leads to new signatures of the oscillatory modes which involve distant devices of the grid called coupling modes in the sequel. Indeed, in the past, these modes, called inter-area modes, were quasi-exclusively due to large thermoelectric synchronous generators of which coherent groups of turbine-generator rotors oscillate one against other (see, e.g., [24]). Recently, other types of

M. Kouki and B. Marinescu are with Ecole Centrale Nantes-LS2N-CNRS, France, e-mails: \{Mohamed.Kouki,Bogdan.Marinescu\}@ec-nantes.fr.

F. Xavier is with RTE-R\&D, France, e-mail: florent.xavier@rte-france.com. coupling modes have been put into evidence: in [1] and [2] coupling modes between classic synchronous generators but related to the electric parts (axes D or Q) of distant generators have been studied. They are thus not of inter-area nature. In [17] oscillatory modes between converters of distant HVDCs were put into evidence and analyzed. Also, power converters (from HVDC or Power Parks Modules) may have important participation in inter-area modes and thus interact with synchronous generators [1], [7], [32]. These new types of modes are called electrical coupling modes in the sequel. Groups of generators which swing together for a given disturbance were constructed based on coherency (see [5], [10], [22], [25] or related references) or synchrony ( [14], [23] and related references). Some hypothesis at the base of these notions like slow coherency/singular perturbation decomposition or dominance of the synchronous generators are no longer valid for the new classes of coupling modes mentioned above.

Next, massive PPM integration (mainly because of new sources of renewable energy) increases the size of the resulting mathematical model which is a main difficulty for modal analysis.

Finally, the approaches should be exhaustive, in the sense that all coupling modes of a given grid should be found. This means a full scan of the system for all types of coupling modes and not only numeric computation of a mode in a vicinity of a given -by some engineering a priori knowledge or intuition - starting point in the frequency plane. Moreover, in cases like investigation of a new grid or interconnection of two existing grids, no a priori knowledge about the structure or shape of the oscillations can be used.

In this paper we develop a new methodology to overcome the difficulties mentioned above. It provides an analytic way to put into evidence all the coupling modes of a given power system by grouping dynamic devices which swing together into coupling groups. Next, the modes of each group are computed based on the Selective Modal Analysis (SMA) approach [21] to finally provide all the coupling modes of the overall system.

The proposed methodology works independently of the system's order. For this, analysis techniques from dynamic system theory are combined with matrix computations both 
adapted for large-scale. Preliminary results were presented in [11].

This paper is organized as follows. Section II provides the problem formulation. Section III gives the details of the proposed method. In Section IV it is shown how the methodology is adapted to work with good performances in large-scale. Section V is devoted to concluding remarks.

\section{Problem FORMUlation}

Usual analytical modeling of a power system leads to the following set of Differential-Algebraic Equations (DAE)

$$
\left\{\begin{array}{l}
\dot{x}=g(x, v) \\
0=h(x, v)
\end{array}\right.
$$

where $x \in \mathbb{R}^{n}$ are the differential variables (angles and speed of the rotating machines, condensers' voltages, state variables of the regulation blocks) and $v \in \mathbb{R}^{q}$ are the algebraic variables (real and imaginary parts of the grid voltages) (see, e.g., [12], [16]). (1) usually contains dynamic models of generators along with their voltage and frequency regulations, of loads and grid representation.

If the algebraic variables are eliminated, (1) leads to a nonlinear autonomous state-space form

$$
\dot{x}=f(x) .
$$

When linearized around an equilibrium (load-flow) point, (2) gives a linear state-space representation

$$
\dot{x}=A x .
$$

where $A \in \mathbb{R}^{n \times n}$. The eigenvalues of the state matrix $A$, called the modes of the system, define - in a linear approximation - the dynamics of the overall system. From the mathematical point of view, there are $n$ such eigenvalues which can be real or complex conjugate. The real ones correspond to aperiodic time responses while the complex conjugate pairs describe oscillatory phenomena. Such oscillatory phenomena may involve several distant dynamic devices - socalled coupling modes in the sequel - like the inter-area modes which are electromechanical oscillations of classic generators. Notice that the inter-area modes are not the only coupling modes of a power system, like discussed in the next paragraph.

\section{A. Coupling modes}

As the PPMs integration in interconnected power systems has significantly increased, inter-area modes are not any more the only existing coupling modes and coexist with the new electrical coupling modes mentioned in the Introduction. For example, in the Spain-France interconnected power system model in Fig. 1 which consists of 23 synchronous generators, a wind farm, a PV solar farm and a HVDC link between France and Spain, 4 types of coupling modes exist.

The first type is an inter-area mode and an example is given in Table X. Indeed, the highest participation parts are the rotors of distant synchronous generators.

The remaining three types are electrical coupling modes and some examples are given in Tables V...IX.

The mode provided in Table VIII involves 15 geographically distant synchronous generators and 3 converters. This mode is an electrical coupling mode because the participation of the electrical parts associated to the D-axes and to the converters are more important than the rotors' participation.

The mode reported in Table IX involves 5 geographically distant synchronous generators. It is not of inter-area nature because the highest participation parts are not associated to the rotors but to D-axes.

The last type of coupling modes is due to the electrical coupling of converters such as the modes reported in Tables $\mathrm{V}, \mathrm{VI}$ and VII. The frequencies of these modes are ranged between $11 \mathrm{~Hz}$ and $28 \mathrm{~Hz}$. They are thus similar to the coupling modes studied in [17].

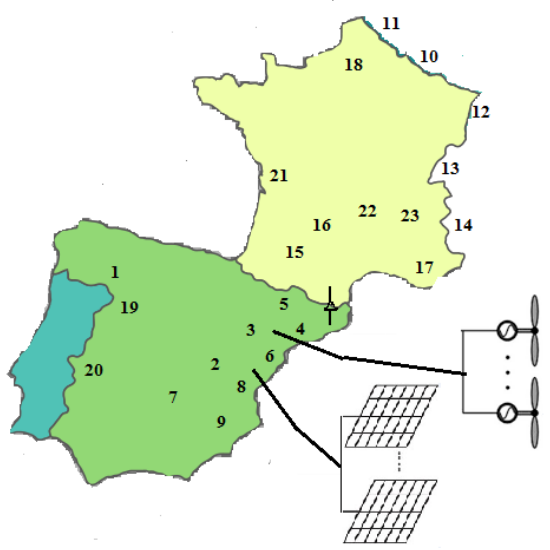

Fig. 1. Spain-France interconnected systems

\section{B. Exhaustive scan of coupling modes}

Modal analysis studies are mainly focused on coupling modes. Notice that not all complex eigenvalues of the state matrix $A$ are related to oscillatory phenomena involving distant devices; some may describe local oscillations related only to one dynamic device (e.g., a generator or a converter) of the system. The latter are easy to put into evidence since one can thus use only the equations related to the dynamic device itself, ignoring the grid equations. As a consequence, the challenge is thus the computation of coupling modes since it is difficult to have for that a purely algebraic computation point of view. Indeed, on the one hand, coupling behaviour should be identified since not all complex modes are of interest and, on the other hand, exhaustive computation of complex modes of a large-scale system is numerically difficult. Moreover, even if the latter would be possible, an analysis of the nature of the found modes is needed a posteriori.

Several approaches to find coupling modes of classic interconnected power systems have been developed and discussed in, e.g., [3], [19], [21], [27]. Although they can be applied in large-scale, such approaches provide only selective modal analysis. This means that only few modes can be found 
in an iterative manner if good initial points and thus some knowledge from the system are available. They are thus difficult to apply to a completely unknown system for which an exhaustive modal analysis, i.e., a full scan for all coupling modes is needed.

In Generalized Selective Modal Analysis (GSMA) [27], initial points are computed in an exhaustive manner but using the so-called classic model for the synchronous generators (i.e., only the swing equation of the turbine-generator unit, see, e.g., [12], [24]). As a consequence, only inter-area electromechanical modes could be found and the case of large-scale power systems with high power electronics penetration is not covered.

The problem is thus to find all the coupling modes of a given power system. The latter may contain any type of dynamic devices, i.e., not only classic synchronous generators but also power electronics. No a priori knowlegde about the structure and dynamic patterns (i.e., shape of oscillations, frequency of the modes, ...) is supposed, so the approach should be able to use only dynamic models (1) and (3). The approach should work for large-scale and be exhaustive in the sense that all the coupling modes should be provided.

\section{PROPOSED METHOD}

We propose here an exhaustive method that systematically quantifies the interaction between the classic synchronous generators, between the converters, and between the converters and classic synchronous generators, in order to find all coupling modes of any given power system. Once these interactions put into evidence, eigencomputations are run on reduced parts of the system which corresponds to devices with coupled behaviour.

A coupling mode quantifies the interaction between two or more distant dynamic devices. This means that for some dynamics excited on a dynamic device, significant responses are registered on distant devices. For a given device, one can thus define a group of devices to which it is coupled. This means that, to some extent, the coupling modes which involve the devices of such group strongly depend on dynamic models of the devices of the group and much less on the dynamic models of the devices outside the group. As a consequence, computation of these modes can be run on the dynamic model of the group and with few information from the rest of the system. This principle is the basis of so-called Selective Modal Analysis (SMA) approaches [3], [21]. The methodology we propose is structured in two steps: first, coupling groups are defined for each dynamic device of the system and, next, SMA is performed for some well-chosen groups till all the coupling modes are found. Classification and redundancy in construction of each group of coupled devices is managed in order to put into evidence and compute all the coupling modes of the system with a minimal computation burden.

\section{A. Coupling groups identification}

1) Inputs-outputs selection: The above definition of the coupling groups corresponds to an input-output view of the power system. For that, starting from (3), one has to choose inputs $u$ and outputs $y$ to obtain an input-output model

$$
\left\{\begin{array}{l}
\dot{x}=A x+B u \\
y=C x
\end{array}\right.
$$

Notice that from (4) one can also extract a transfer function $H(s)=C(s I-A)^{-1} B$. Inputs $u$ must be chosen as the ones which excite one or several coupling modes and outputs $y$ must be the variables of distant devices with significant responses in such cases. Roughly speaking, this means that the considered coupling modes are dominant in the transfer $H(s)$ above. From the system point of view, this means that the considered coupling modes are controllable by $u$ and observable from $y$ (see [24] or another basic textbook for these technical but basic notions). It is well known (see, e.g., [24]) that, the inter-area modes, are controllable from the references of the voltage regulators $V_{\text {ref }}$ and observable from the speed of each synchronous generator $\omega$. Thus, $u=V_{\text {ref }}$ and $y=\omega$ in this case. For the case of converters - stand alone or connecting generators to the grid -, similar observability/controllability evaluations and several trials let us conclude that transfer between the reference of the voltage regulation loop and the (active or reactive) power is the most suited.

Intuitively, this can be viewed as follows: if a coupling mode was known, $u$ and $y$ would be selected (by physical/system considerations above) such that transfer $H(s)$ is mainly given by that dynamic (the coupling mode). But these modes are not a priori known and the target is to put them into evidence. For that, the reasoning is reversed: all the potentially candidates transfer functions $H(s)$ are checked for their coupling dynamics. If such coupling behaviours are put into evidence, (dominant) modes will be next computed and this will lead to coupling modes. At this stage of the procedure, candidates for inputs $u$ and outputs $y$ have been given. Next step consists in detecting coupling behaviour between these inputs and outputs in order to form the coupling groups of generators and power electronic devices mentioned above.

2) Device selection by simulation: The most intuitive way to settle the coupling groups is by simulation. This means to excite each dynamic device $i$ of the interconnected power system $(i=1, \ldots, n$, where $n$ is the number of dynamic devices) with the temporal signals defined above $\left(u_{i}\right)$. Next, a correlation analysis between $u_{i}$ and the outputs $y_{j}, j=1, \ldots, n$ of all dynamic devices is carried out.

An example of Spain-France system groups obtained with this technique is provided in Table I [11]. The threshold used for the correlation analysis was $1.8 \%$. Each row gives the groups of the corresponding device. For example, generator G1 of row 1 of the table is found to be coupled with generators G9, G19, G20, and G21 but not coupled with the other dynamic devices.

Although this technique is intuitive, it can be fastidious in case of large-scale systems. Also, it has the disadvantage not to be analytic as it relays on simulations. It is also 
TABLE I

Groups Generated By the Simulation teChNique

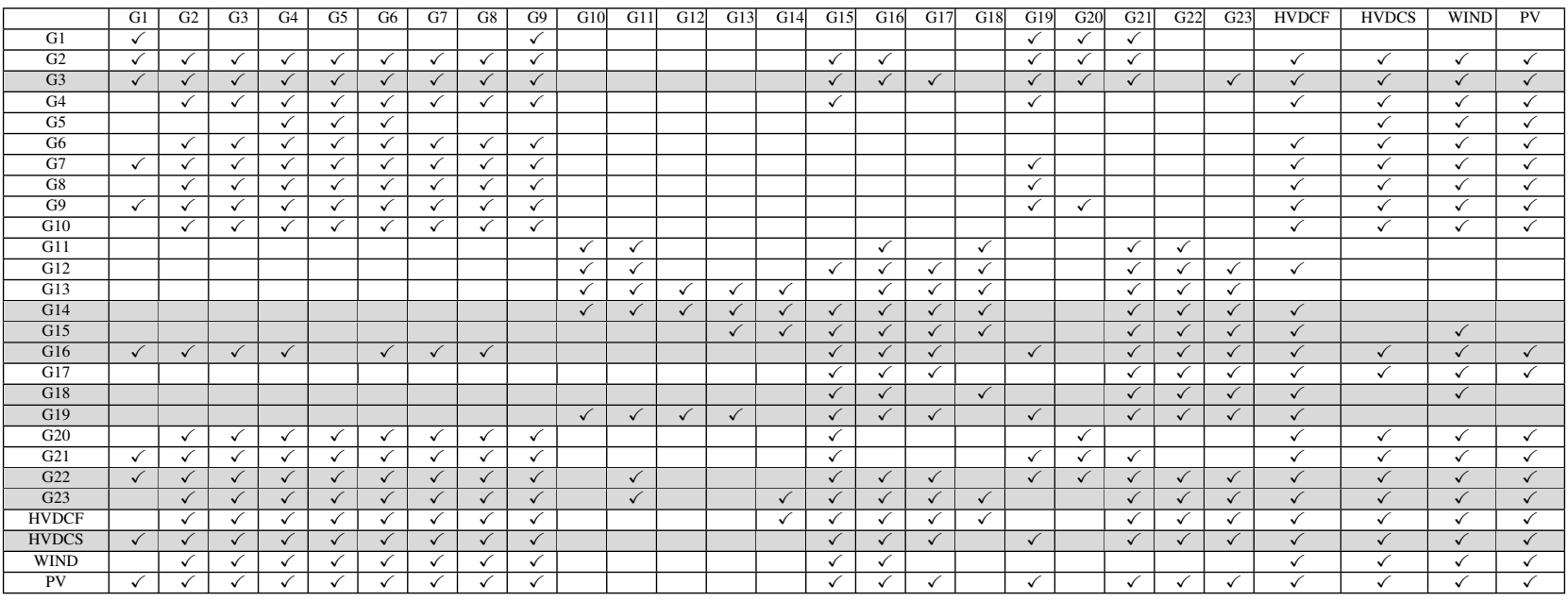

a single-input/single-output (SISO) technique. For these reasons, the following alternative was proposed.

3) Analytic device selection: Interactions between the dynamic devices of a given power system can be quantified analytically in several ways starting from a state-space realization (4) of the system. Indeed, as high modal controllability and observability measures denote high coupling degree between input and output, these measures can be used to select the most coupled outputs $y_{j}, j=1, \ldots, n$ with the given input $u_{i}$.

Among several interaction indexes that were developed and used in the control design domain (such as Relative Sensitivity Technique [8], [33], Participation matrix [6], and Hankel Interaction Index Array [31]), the Relative Sensitivity Technique (RST) is used here since it is applicable to large-scale systems.

RST quantifies the interaction sensitivity between the inputs $u_{i}$ and the outputs $y_{j}$. Indeed, it assesses the magnitude of the response of the $j^{t h}$ output to an impulse on input $i$ using the relative degree. The latter is a well-known measure of physically closeness between an input and an output of a dynamic system [4], [33]. More precisely, it provides valuable information on how directly a manipulated input can affect an output variable. Mathematically, it is defined as the lower integer $r_{i j}$ such that

$$
\begin{cases}C_{j} A^{r_{i j}-1} B_{i} & \neq 0 \\ & \text { or } \\ r_{i j} & =\infty \text { if such integer does not exist }\end{cases}
$$

According to the above relative degree definition, the sensitivity matrix is

$$
s_{i j}=C_{j} A^{r_{i j}-1} B_{i} \Rightarrow S=\left[\begin{array}{ccc}
s_{11} & \ldots & s_{n m} \\
\vdots & \ldots & \vdots \\
s_{m 1} & \ldots & s_{m n}
\end{array}\right]
$$

Based on $S$, the groups of coupled dynamic can be gener- ated according to two approximations.

The first one consists to normalize the sensitivity matrix $S$ according to the Euclidean norm [8]. Hence, the final Relative Sensitivity Matrix $(R S M)$ is obtained

$$
R S M_{i j}=\frac{s_{i j}^{2}}{\sum_{i=1}^{n} s_{i j}^{2}},
$$

where $R S M_{i j} \in[0,1]$.

Closer $R S M_{i j}$ is to one, more the $j^{\text {th }}$ output is sensitive to the $i^{\text {th }}$ input, and hence the modal controllability and observability measures associated to the dynamic devices ( $i$ and $j$ ) are high. This means that $(i, j)$ is an appropriate input-output pair and $j$ can be taken for an element of the group $i$.

For the second approximation, the selection of the appropriate input-output pair $(i, j)$ can be refined by considering also the effect of the other pairs $\left(i^{\prime}, j^{\prime}\right), i^{\prime} \neq i, j^{\prime} \neq j$ [33]. Mathematically, the $R S M$ is, in this case, computed as

- All other pairs are open: $u_{k}=0 \forall k \neq j$ then

$$
[S]_{i j}=\left(\frac{\partial y_{i}}{\partial u_{j}}\right)_{u_{k}=0, k \neq j}
$$

- All other pairs are closed: $y_{k}=0 \forall k \neq i$ then

$$
\left[S^{-1}\right]_{j i}=\left(\frac{\partial u_{j}}{\partial y_{i}}\right)_{y_{k}=0, k \neq i}
$$

From (8) and (9), the relative sensitivity is

$$
R S M_{i j}=[S]_{i j}\left[S^{-1}\right]_{j i}
$$

An example of computation of $R S M$ matrix is given in Appendix A.

Identification of the groups of coupled dynamic devices (appropriate input-output pairs) of the grid using the absolute value of $R S M$ is done according to a chosen threshold $\delta$ :

- $R S M_{i j}<\delta \Rightarrow$ device $i$ and device $j$ are not coupled,

- $R S M_{i j} \geq \delta \Rightarrow$ device $i$ and device $j$ are coupled.

Table II gives the groups obtained with the second RST approximation for the Spain-France system with $\delta=0.02 \%$. 
These groups are almost similar to those obtained by the simulation technique (Table I). This validates the RST groups construction against the simulation techniques.

Several groups of Table I and Table II are quasi similar. It is obvious that their analysis would give almost the same results. For this, only the groups indicated in gray on Tables I and II are used. Here, the final groups selection is manually operated, and it is adapted in section IV for the large-scale systems' case.

Notice that the selection of the threshold $\delta$ as well as the correlation threshold for the simulation technique is heuristic and related to the method used in groups identification and to the system features. In general, it has to be small in order to ensure high redundancy in the construction of groups. Therefore, for each group a maximum number of modes will be found and, in the end, the eigencalculation is surely exhaustive in the sense that all the coupling modes of the overall power system will be found.

For both simulation and analytic selection methods, the resulting matrices (Tables I and II) are almost but not precisely symmetric. This is due to the way in which the couplings are tracked: in row $i$ device $i$ is displayed coupled with device $j$ if input $u_{i}$ strongly excites a dynamics/mode which is highly observable on output $j$ of device $i$. This does not necessarily means that the input $u_{j}$ on device $j$ would provide significant output on $y_{i}$ of device $i$. However, relation is bilateral in most of the cases. For example in Table I, such bilateral groups of devices are $\left\{G_{2}, G_{3}, G_{6}, G_{7}, G_{8}, G_{9}\right\},\left\{G_{15}, G_{16}, G_{17}\right\}$, $\left\{G_{13}, G_{14}\right\},\left\{G_{22}, G_{23}\right\},\left\{H V D C_{F}, H V D C_{S}\right.$, Wind, $\left.P V\right\}$. Symmetry is not a target since, as mentioned before, redundancy is needed in definition of these groups at this stage. This will be exploited in Section III.C to exhaustively compute the coupling modes along with a strategy to avoid repetition in computation.

Notice also that, in contrast with the simulation procedure presented in section above, this approach is also direct multiinput/multi-output (MIMO).

At this stage, groups of dynamic devices have been formed. Next step of the approach consists in modal computation. This is done for each formed group and neglecting the rest of the system. This is possible because the groups formed have strongly connected internal dynamics and few connections with the rest of the grid. SMA is a method which exploits this property to run eigencalculation on the group only and neglecting the rest of the system. This is a key point in burden computation reduction for the approach we propose. Groups forming and eigencalculations are thus two distinct parts of the overall methodology and the latter uses the inputs from the first one.

\section{B. Modes computation}

For each coupling group an approximation of all its modes can be performed using SMA. SMA is an efficient iterative approach for analyzing only a selected part of large-scale state linear systems $(\dot{x}=A x)$ [21]. Main results used in the sequel are briefly recalled here. The reader should check [21] and related references for details. The selected part should have small interaction with the rest of the system. Indeed, construction of the coupling groups lays on this principle. For that, the state vector $x$ is divided into two parts, a relevant part $r$ which corresponds in our case to the studied coupling group (i.e., $r$ contains the states of the dynamic devices of the considered coupling group), and a less relevant part $z$ which is the rest of the state vector of the overall system:

$$
\dot{x}=\left[\begin{array}{c}
\dot{r} \\
\dot{z}
\end{array}\right]=\left[\begin{array}{ll}
A_{r r} & A_{r z} \\
A_{z r} & A_{z z}
\end{array}\right]\left[\begin{array}{l}
r \\
z
\end{array}\right],
$$

with $r \in \mathbb{R}^{q}, z \in \mathbb{R}^{m-q}$ and $m$ is the length of $x$. This corresponds to Fig.2

The effect of the less relevant parts on the relevant part can

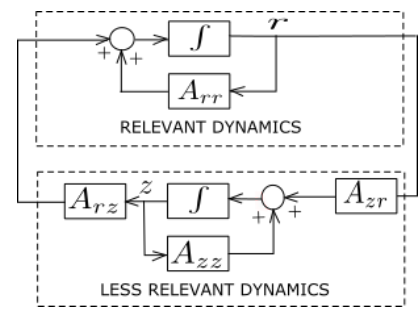

Fig. 2. Block diagram representation of the separation between the relevant and less relevant parts in SMA approach

be taken into account in a simplified way as in Fig.3 where $\mathbf{M}$ is the transfer matrix of the less relevant dynamics which corresponds to the eigenvalues of interest. The latter along with their associated right eigenvectors are generated at $k^{t h}$ iteration for each coupling group

$$
{ }^{k+1} \mathbf{M}\left[{ }^{k} v_{1} \ldots{ }^{k} v_{r}\right]=\left[H\left({ }^{k} \lambda_{1}\right){ }^{k} v_{1} \ldots H\left({ }^{k} \lambda_{r}\right)^{k} v_{r}\right],
$$

where ${ }^{k} v_{1}$ and $H\left({ }^{k} \lambda_{1}\right)=A_{r z}\left({ }^{k} \lambda_{1} I-A_{z z}\right)^{-1} A_{z r}$ are the right eigenvector and the transfer function of the less relevant dynamics associated to the eigenvalue of interest ${ }^{k} \lambda_{1}$, respectively.

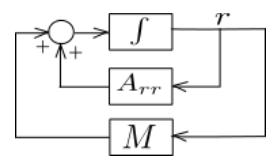

Fig. 3. Block diagram of the transfer matrix $M$ integration with the relevant part

As illustrated in Table III, SMA computation on the groups of Spain-France system gives an overall good approximation of most of the coupling modes except very few ones. For example, compared to the exact values (column 2 of Table III) of the modes computed directly on the overall system (with standard methods like, e.g., Arnoldi [3]), mode 21 was exactly approximated which was not the case for the mode 4 .

Notice that the size of $A_{r r}$ is much smaller than the one of $A$ so that computation of its all modes is possible by standard approaches (like, e.g., the eig function in Matlab). For example, for the large-scale system presented in Section $\mathrm{IV}$, the largest coupling group contains 341 dynamic devices. 
TABLE II

GROUPS GENERATED BY RST

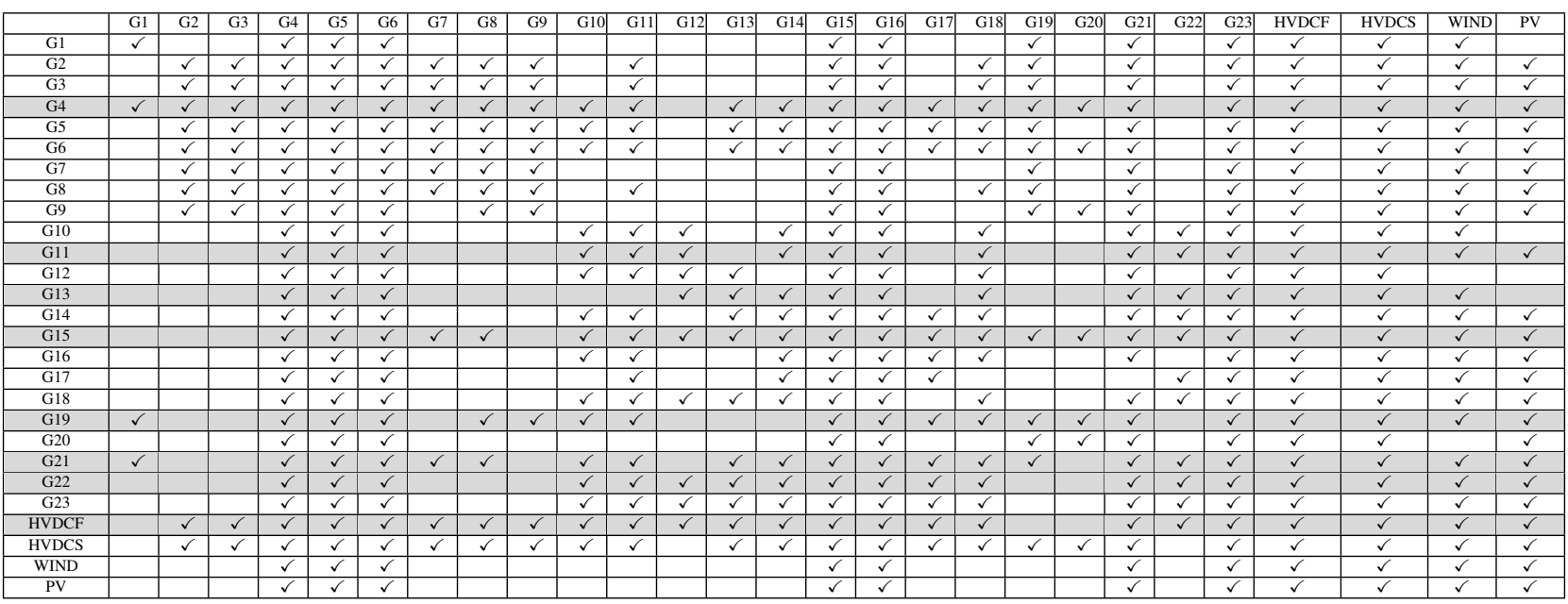

TABLE III

COMPARISON BETWEEN PROPOSED METHODOLOGY, SIMULATION TECHNIQUE AND FULL MODEL

\begin{tabular}{|c|c|c|c|}
\hline Number & Mode of full model & Mode with New Methodology & Mode with Simulation \\
\hline 1 & $-131.5163+j^{*} 178.9952$ & $-131.5181+j * 178.9839$ & $-131.5123+\mathrm{j} * 178.9706$ \\
\hline 2 & $-135.0671+j^{*} 164.8829$ & $-134.7647+j * 167.6751$ & $-136.7753+j^{*} 156.8727$ \\
\hline 3 & $-139.8447+\mathrm{j}^{*} 136.6876$ & $-138.7261+j^{*} 140.7270$ & $-138.2738+j^{*} * 144.4395$ \\
\hline 4 & $-144.6338+\mathrm{j} * 97.2166$ & $-141.090+j^{*} 112.3975$ & $-140.7495+\mathrm{j}^{*} 129.5819$ \\
\hline 5 & $-73.1979+\mathrm{j}^{*} 72.7548$ & $-73.1989+j^{*} 72.7587$ & $-73.1998+\mathrm{j}^{*} 72.7540$ \\
\hline 7 & $-17.7045+\mathrm{j} * 55.9061$ & $-17.7045+j * 55.9061$ & $-17.7045+j^{*} 55.9061$ \\
\hline . & & . & 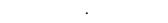 \\
\hline 21 & $-0.3808+j^{*} 6.0187$ & $-0.3808+j^{*} 6.0188$ & $-0.3809+j^{*} 6.0187$ \\
\hline 22 & $-0.3001+j * 5.5004$ & $-0.3078+j * 5.5097$ & $-0.3145+j * 5.4447$ \\
\hline 23 & $-0.5725+j^{*} 4.9878$ & $-0.5726+j^{*} 4.9877$ & $-0.5725+j^{*} 4.9876$ \\
\hline 33 & $-0.2751+\mathrm{j}^{*} 1.0169$ & $-0.2708+\mathrm{j}^{*} 1.0059$ & $-0.2799+\mathrm{j}^{*} 1.0591$ \\
\hline 35 & $-1.4482+j^{*} 0.8053$ & $-1.5563+j^{*} 0.7389$ & $-1.1150+j^{*} 0.9370$ \\
\hline . & . & . & \\
\hline 51 & $-0.2248+\mathrm{j}^{*} 0.1895$ & $-0.2248+j^{*} 0.1895$ & $-0.2248+j^{*} 0.1895$ \\
\hline 59 & $-0.1236+j^{*} 0.0601$ & $-0.1225+j * 0.0602$ & $-0.1232+j^{*} 0.0605$ \\
\hline
\end{tabular}

The resulting dimension of $A_{r r}$ is 6277 which is still feasible.

The errors between the modes that were not precisely computed and their exact values are very small. This means that the coupling groups computation presented above is efficient in the sense that there are no major interactions between the dynamic devices of each of these groups and the rest of the system.

However, the few approximation errors can be overcome as shown below.

\section{Exact and exhaustive modal analysis}

First, the coupling modes found by SMA for each coupling group should be adjusted to the exact values of the coupling modes of the overall system. For this, any iterative classic selective modal analysis like e.g., modified Arnoldi or GSMA [3] method can be used with the approximative values found by SMA as initial points.

An example showing the high accuracy provided by the modified Arnoldi method is given in Table IV for the coupling modes of Spain-France system. The results illustrated in Table IV are reported for modes 2 and 3 and this shows that the small errors that might exist (as explained in the end of preceding section) can thus be systematically eliminated.

Next, modal analysis should be exhaustive in the sense that all the coupling models of the overall system should be found. For that, it is sufficient to apply SMA on all coupling groups. However, as the latter groups were constructed with some redundancy as explained in Section III-A3, all the coupling modes of the overall system will be found before treating all the groups. Indeed, on the France-Spain system, only 8 groups are sufficient.

Found modes are analyzed in Appendix B where the types discussed in Section II are put into evidence. Notice that all found modes, i.e., the ones in Table III are proved to be coupling modes by computation of participation factors. This proves that coupling detection by RST technique adopted in Section III.A, which is a well known technique in automatic control, is also efficient for the specificities of power systems. Same analysis/check is carried in Appendix C for the results obtained on the large-scale representation of the European system used in Section IV.

The main steps of the proposed approach are summarized in Algorithm 1.

Algorithm 1 provides all the coupling modes if the eigenvalues generated in step 6.3.3 are quite similar to eigenvalues of the matrix $A$. Numerically, this condition is implemented 
TABLE IV

EXACT Computation of THE RELEVANT DyNAMics

\begin{tabular}{|l|c|c|c|}
\hline Number & Mode with Arnodi method & Mode with New Methodology & Mode with Simulation \\
\hline 2 & $-135.0671+\mathrm{j}^{*} 164.8829$ & $-134.7647+\mathrm{j}^{*} 167.6751$ & $-136.7753+\mathrm{j}^{*} 156.8727$ \\
\hline 3 & $-139.8447+\mathrm{j}^{*} 136.6876$ & $-138.7261+\mathrm{j}^{*} 140.7270$ & $-138.2738+\mathrm{j}^{*} 144.4395$ \\
\hline
\end{tabular}

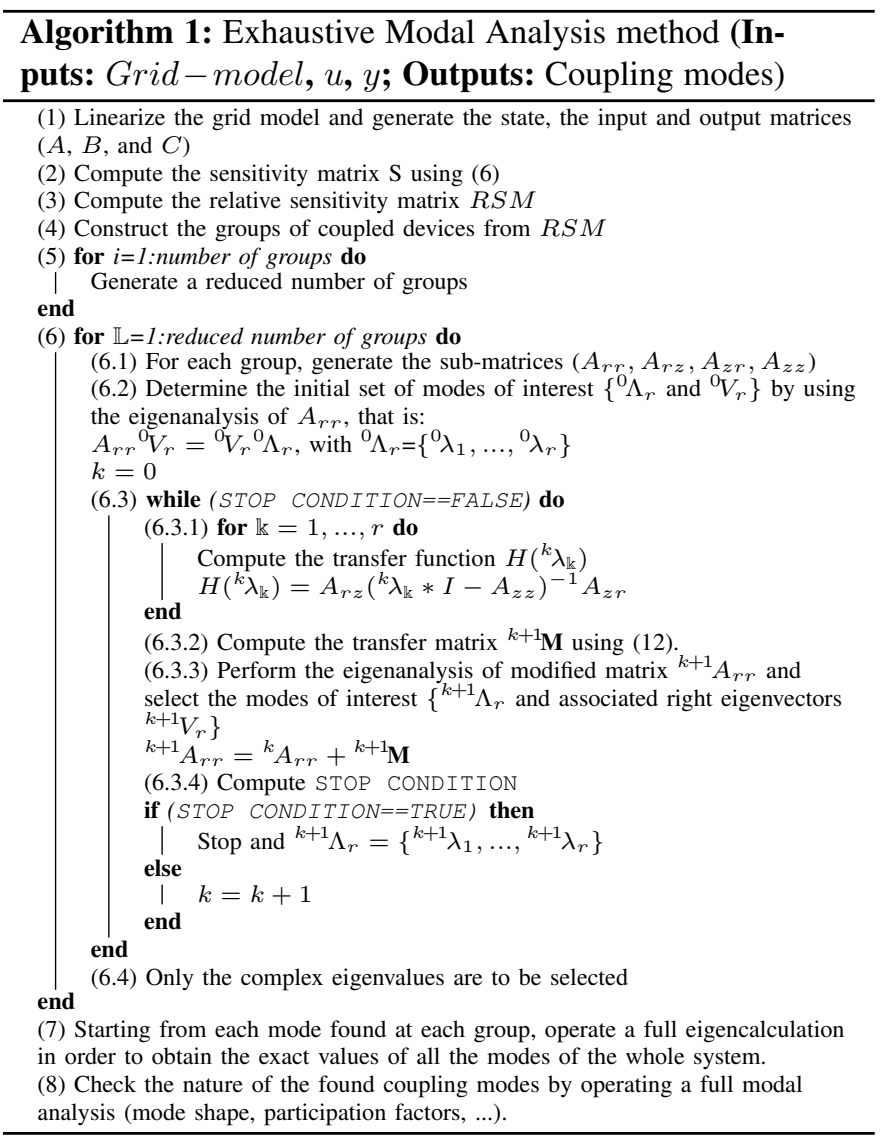

here as

$$
\operatorname{cond}\left({ }^{k+1} \lambda_{\mathbb{k}} * I-A\right) \geq \epsilon,
$$

where $\operatorname{cond}(M)=\frac{\sigma_{\max }(M)}{\sigma_{\min }(M)}$ is the condition number of matrix $M$, with $\sigma_{\max }$ the highest singular value of $M$ and $\sigma_{\text {min }}$ its lowest singular value (see, e.g., [9]).

Theoretically, for ${ }^{k+1} \lambda_{\mathbb{k}}$ to belong to the $A$ spectrum, $\operatorname{cond}\left({ }^{k+1} \lambda_{\mathbb{k}} * I-A\right)$ should tend to $\infty$ (see, e.g., [9]). In practice, it is sufficient for $\operatorname{cond}\left({ }^{k+1} \lambda_{\mathbb{k}} * I-A\right)$ to be greater/or equal to a threshold $\epsilon$. For example, the threshold used in validating the eigenvalues of the Spain-France interconnected power system is equal to $10^{3}$. Indeed, for lager values some modes are missed. This threshold is deduced from trial and errors and seems valid for medium-scale power systems. Specific implementation for large-scale is presented in the next section.

\section{APPLICATION TO LARGE-SCALE POWER SYSTEMS}

\section{A. Specific implementation}

If two or several coupling groups consist of almost the same dynamic devices, it is obvious that their SMA analysis would provide almost the same coupling modes. This remark allows one to reduce the number of groups to be considered in case of large-scale systems.

Furthermore, the stop condition (step 6.3.4 Algorithm 1) is computationally heavy in large-scale cases. One way to alleviate computations is thus suppress it and require a prefixed number of iterations $k$. This will lead to rapid computation of all relevant dynamics of the considered coupling group. The price to pay is twofold: first, the obtained eigenvalues are not all exact and, next, some of them could not be approximations of eigenvalues of $A$. First point is not critical if $k$ is sufficient high in order to obtain good enough approximations as starting points for step 7 of Algorithm 1. Indeed, the iterative eigencomputation on the full system (matrix $A$ ) will in this case converge. The second point is also not critical as bad starting points for step 7 computed at this stage will lead to bad convergence of step 7 and with no incidence of the found modes.

\section{B. Validation on a large-scale power system}

The above implementation is tested in large-scale on a detailed representation of the full European power system including the Turkey zone introduced in [13]. Known as the ENTSO-E Dynamic Reference Model (DRM), this model is actually used in Europe for dynamic analysis which involves cross-border phenomena.

It consists of 1074 generators, 7652 buses, 10465 lines and 2550 transformers and the resulting order of the state is 16784. The exhaustive modal analysis of this model using the proposed method provided 5800 modes using fixed iterations number $k=1$ and only 66 groups. This reduced number of groups corresponds to a redundancy selection $50 \%$ and the initial number of groups was equal to 1074 .

The nature of the found modes is given in Appendix C.

\section{Conclusions}

In this paper, we proposed a new methodology that is able to find all coupling modes of a given power system. This methodology does not require any a priori knowledge neither about the nature of modes nor about the participating dynamic devices. Research is guided to the coupling behaviours and this avoids random numerical computations and thus guarantees/speedsup exhaustive analysis. These are the two main advantages against existing methods which focus on inter-area modes of the system and rely on hypotheis that such modes involve mainly large classic synchronous generators.

This methodology enables to directly consider any kind of dynamic devices of the power system like, e.g, Power Park 
Modules and not only classic synchronous generators. It is also analytic in the sense that involves only computations of the matrices of the system without any need for manual operations like dynamic simulations. It can thus be integrated in numerical tools or operational platforms.

Approach is based on matrix computation and dynamic system analysis implemented for large-scale application. Systems like the European interconnected power system were treated.

Main target is the scan of the system for all coupling modes to put into evidence oscillations, but the real and complex modes that do not involve distant devices can be easily computed from the equations of each dynamic device individually (i.e., ignoring the grid connections).

Forthcoming publications will give more details about results of the scan of large-scale power systems.

\section{APPENDIX A}

\section{RELATIVE SENSITIVITY EXAMPLE}

Consider a second order linear system, i.e., $A \in \mathbb{R}^{2 * 2}$, $B \in \mathbb{R}^{2 * 1}$ and $C \in \mathbb{R}^{1 * 2}$. Let

$$
S=\left[\begin{array}{ll}
s_{11} & s_{12} \\
s_{21} & s_{22}
\end{array}\right]
$$

be the sensitivity matrix (3). Then

$$
\begin{aligned}
& y_{1}=s_{11} u_{1}+s_{12} u_{2}, \\
& y_{2}=s_{21} u_{1}+s_{22} u_{2} .
\end{aligned}
$$

If all other pairs are open $\left(u_{2}=0\right.$ and (5)):

$$
\underbrace{y_{1}=s_{11} u_{1}}_{\text {main pair interaction }} \text {. }
$$

If all other pairs are closed $\left(y_{2}=0\right.$ and (6)):

$$
u_{2}=\frac{-s_{21}}{s_{22}} u_{1} \text {. }
$$

From (15) and (18) follows

$$
\underbrace{y_{1}=\left(s_{11}+\frac{-s_{21}}{s_{22}} s_{12}\right) u_{1}}_{\text {interaction of other pairs }}
$$

and the final RSM matrix is

$$
R S M=S \otimes\left(S^{-T}\right)=\left[\begin{array}{ll}
R S M_{11} & R S M_{12} \\
R S M_{21} & R S M_{22}
\end{array}\right]
$$

with

$$
\begin{array}{r}
R S M_{11}=\frac{\text { Open }- \text { pair interaction magnitude }\left(\text { with } u_{2}=0\right)}{\text { Closed }- \text { pair interaction magnitude }\left(\text { with } y_{2}=0\right.} \\
=\frac{1}{1-\frac{s_{12} s_{21}}{s_{11} s_{22}}} .
\end{array}
$$

If $\frac{s_{12} s_{21}}{s_{11} s_{22}}$ converges to 0 , then $R S M_{11}$ converges to 1 . Therefore the $j^{\text {th }}$ output is strongly sensitive to the $i^{\text {th }}$ input and the dynamic device $i$ is strongly coupled to the dynamic device $j$.
TABLE V

MACHINES WITH HIGHEST PARTICIPATION IN MODE 1

\begin{tabular}{|c|c|c|}
\hline Mac. & Rel. Part. (\%) & Phase r. evec. $\delta$ (deg) \\
\hline Wind & 100 & 0.0 \\
PV & 78.5 & 0.0 \\
HVDC & 29.9 & 0.0 \\
\hline
\end{tabular}

TABLE VI

MACHINES WITH HIGHEST PARTICIPATION IN MODE 4

\begin{tabular}{|c|c|c|}
\hline Mac. & Rel. Part. (\%) & Phase r. evec. $\delta$ (deg) \\
\hline HVDC & 100 & 0.0 \\
Wind & 32.4 & 0.0 \\
PV & 18.9 & 0.0 \\
\hline
\end{tabular}

\section{APPENDIX B \\ Modes of SPAIN-France POWER SYSTEM}

Details of the base-case modes of Spain-France power system are given in Tables V...X.

From the participation factors shown in Tables V, VI and VII, one can conclude that modes 1,4 and 5 are electrical coupling modes of power converters in the frequency range $[11 \mathrm{~Hz} 28.52 \mathrm{~Hz}]$.

Table VIII gives the results (sub-participation factors, phases,...) of the coupling mode number 35. It involves 15 geographically distant synchronous generators and 3 converters. The contribution of electrical dynamics is more important than the rotor contribution. The electrical contribution is related to the D-axes and to converters parts. Thus, it is an electrical coupling mode between converters and classic synchronous generators.

The results of mode 33 are provided in Table IX. It involves 5 geographically distant synchronous generators. The contribution of electrical dynamics related to D-axes is more important than the rotor contribution. It is thus an electrical coupling mode between synchronous generators but not of inter-area nature.

The results of mode 22 are given in Table X. Notice that rotors have the highest participation. Also column 5 shows a phase opposition of right eigenvectors associated to the speed deviations of the most participating generators. It is thus a

TABLE VII

MACHINES WITH HIGHEST PARTICIPATION IN MODE 5

\begin{tabular}{|c|c|c|}
\hline Mac. & Rel. Part. (\%) & Phase r. evec. $\delta$ (deg) \\
\hline Wind & 42.8 & 0.0 \\
PV & 100 & 0.0 \\
\hline
\end{tabular}

TABLE VIII

MACHINES WITH HIGHEST PARTICIPATION IN MODE 35

\begin{tabular}{|c|c|c|c|c|c|}
\hline Mac. & Rel. Part. (\%) & $\begin{array}{c}\text { Phase r. evec. } \delta \\
\text { (deg) }\end{array}$ & Rot. part & $\begin{array}{c}\text { D-axes } \\
\text { part. }\end{array}$ & $\begin{array}{c}\text { Converter } \\
\text { part. }\end{array}$ \\
\hline G20 & 30.2 & -150.5 & 0.0018 & 0.1170 & - \\
G9 & 1.4 & -9.0 & 0.0015 & 0.0035 & - \\
G2 & 76.4 & -0.1 & 0.0109 & 0.4641 & - \\
G3 & 76.4 & -0.1 & 0.0111 & 0.4652 & - \\
G12 & 1.9 & 169.7 & 0.0062 & 0.0128 & - \\
G13 & 2.8 & 168.9 & 0.0100 & 0.01960 & - \\
G18 & 1.1 & 165.9 & 0.0015 & 0.0054 & - \\
G14 & 1.5 & 156.1 & 0.0065 & 0.0016 & - \\
G16 & 1.9 & 66.3 & 0.0001 & 0.0083 & - \\
G21 & 4.3 & 47.2 & 0.0111 & 0.0034 & - \\
G15 & 1.3 & 38.3 & 0.0055 & 0.0001 & - \\
G4 & 1.6 & 2.9 & 0.0012 & 0.0109 & - \\
G6 & 4.4 & 0.6 & 0.0052 & 0.0260 & - \\
G7 & 1.6 & 0.6 & 0.0028 & 0.0065 & - \\
Wind & 3.3 & 0.0 & - & - & 0.0126 \\
HVDC & 5.4 & 0.0 & - & - & 0.0203 \\
PV & 7.3 & 0.0 & - & - & 0.0276 \\
G8 & 100 & 0.0 & 0.0087 & 0.4082 & - \\
\hline
\end{tabular}


TABLE IX

MACHINES WITH HIGHEST PARTICIPATION IN MODE 33

\begin{tabular}{|c|c|c|c|c|}
\hline Mac. & Rel. Part. (\%) & $\begin{array}{c}\text { Phase r. evec. } \delta \\
\text { (deg) }\end{array}$ & Rot. part & $\begin{array}{c}\text { D-axes } \\
\text { part. }\end{array}$ \\
\hline G1 & 25.7 & 0.0 & 0.0079 & 0.1594 \\
G20 & 22.8 & -4.5 & 0.0102 & 0.1488 \\
G19 & 100 & -8.0 & 0.3726 & 0.7138 \\
G13 & 6.5 & -165.6 & 0.0794 & 0.0352 \\
G12 & 4.5 & -165.7 & 0.0538 & 0.02296 \\
\hline
\end{tabular}

TABLE $X$

MACHINES WITH HIGHEST PARTICIPATION IN MODE 22

\begin{tabular}{|c|c|c|c|c|c|}
\hline Mac. & Rel. Part. (\%) & $\begin{array}{c}\text { Phase r. evec. } \delta \\
\text { (deg) }\end{array}$ & Rot. part & $\begin{array}{c}\text { D-axes } \\
\text { part. }\end{array}$ & $\begin{array}{c}\text { Converter } \\
\text { part. }\end{array}$ \\
\hline G8 & 88.6 & 8.2 & 0.2628 & 0.0082 & - \\
G2 & 33.2 & 6.8 & 01002 & 0.0025 & - \\
G3 & 33.1 & 6.8 & 0.0999 & 0.0024 & - \\
G5 & 3.6 & 4.0 & 0.0105 & 0.0001 & - \\
G9 & 28.0 & 3.7 & 0.0826 & 0.0611 & - \\
G4 & 3.1 & 0.4 & 0.0084 & 0.0002 & - \\
HVDC & 1.2 & 0.0 & - & - & 0.0035 \\
G7 & 100 & 0.0 & 0.2957 & 0.0065 & - \\
G6 & 11.1 & -7.3 & 0.0315 & 0.0011 & - \\
G17 & 1.1 & -117.7 & 0.0035 & 0.0002 & - \\
G20 & 2.7 & -128.7 & 0.0101 & 0.0019 & - \\
G16 & 2.3 & -150.2 & 0.0077 & 0.0001 & - \\
G23 & 4.0 & -159.2 & 0.0127 & 0.0007 & - \\
G19 & 19.3 & -161.7 & 0.0648 & 0.0040 & - \\
\hline
\end{tabular}

classic inter-area mode.

\section{APPENDIX C \\ Modes of European Power System}

Some categories of modes were put into evidence on the European system. Some of them are given in Tables XI and XII

TABLE XI

BASE-CASE OF COUPLING MODES

\begin{tabular}{|l|c|c|}
\hline & Mode & Nature of Coupling mode \\
\hline$\lambda_{1}$ & $-0.1247+\mathrm{j} * 1.1142$ & Inter-area mode \\
$\lambda_{2}$ & $-0.44+\mathrm{j} * 9.8714$ & Local mode \\
$\lambda_{3}$ & $-1.5567+\mathrm{j} * 1.3201$ & Electrical mode (D-axe) \\
$\lambda_{4}$ & $-15.8455+\mathrm{j} * 8.7828$ & Electrical mode (Q-axe) \\
\hline
\end{tabular}

TABLE XII

Characteristics OF BASE-CASE COUPLING MOdes

\begin{tabular}{|c|c|c|c|c|c|}
\hline Mode & $\mathrm{f}(\mathrm{Hz})$ & Rot. part & D-axes part. & Q-axes part. & Domi. Mac. \\
\hline$\lambda_{1}$ & 0.177 & 0.0199 & 0.0017 & 0.0001 & 585 \\
$\lambda_{2}$ & 1.571 & 0.1225 & 0.0197 & 0.0632 & 6 \\
$\lambda_{3}$ & 0.1958 & 0.0324 & 0.2711 & 0.0812 & 4 \\
$\lambda_{4}$ & 1.398 & 0.0405 & 0.0258 & 0.5333 & 2 \\
\hline
\end{tabular}

\section{REFERENCES}

[1] L. Arioua and B. Marinescu, "Multivariable control with grid objectives of an HVDC link embedded in a large-scale AC grid", Int. J. Elec. Power, vol. 72, pp. 99-108, 2015.

[2] L. Arioua and B. Marinescu, "Robust grid-oriented control of high voltage DC links embedded in an AC transmission system", Int. J. Robust Nonlin., vol. 26, no. 9, pp. 1944-1961, 2016.

[3] J. Barquin, L. Rouco and H. R. Vargas, "Generalized selective modal analysis", IEEE Power Eng. Soc., vol. 2, pp. 1194-1199, 2002.

[4] E. Bristol, "On a new measure of interaction for multivariable process control”, IEEE Trans. Autom. Control, vol. 11, no. 1, pp. 133-134, 1966.

[5] J. H. Chow, R. Galarza, P. Accari and W. W. Price, "Inertial and slow coherency aggregation algorithms for power system dynamic model reduction", IEEE Trans. Power Syst., vol. 10, no. 2, pp. 680-685, 1995.

[6] A. Conley and M. E. Salgado, "Gramian based interaction measure", in Proc. IEEE 39th Conf. Decis. Contr. P., pp. 5020-5022, Sydney, NSW Australia, 12-15 Decembre, 2000.

[7] S. Eftekharnejad, V. Vittal, G. T. Heydt, B. Keel and J. Loehr, "Small signal stability assessment of power systems with increased penetration of photovoltaic generation: A case study", IEEE Trans. Sustain. Energy, vol. 4, no. 4, pp. 960-967, 2013.
[8] M. Ellis and P. D. Christofides, "Selection of control configurations for economic model predictive control systems", AIChE J., vol. 60, no. 9, pp. 3230-3242, 2014.

[9] G.H. Golub and C.F. Van Loan, Matrix Computations, 4th edition, Johns Hopkins University Press, 2013.

[10] A. M. Khalil and T. Reza, "A dynamic coherency identification method based on frequency deviation signals", IEEE Trans. Power Syst., vol. 31, no. 3, pp. 1779-1787, 2016.

[11] M. Kouki, B. Marinescu and F. Xavier, "Eigencalculation of Coupling Modes in Large-Scale Interconnected Power Systems with High Power Electronics Penetration", in Proc. IEEE 20th Power Sys. Comput. Conf., pp. 1-7, Dublin, Ireland, 1-7 June, 2018.

[12] P. Kundur, N. J. Balu and M. G. Lauby, Power system stability and control, New York: McGraw-hill, 1994.

[13] M. Luther, I. Biernaka and D. Preotescu, "Feasibility Aspects of a Synchronous Coupling of the IPS/UPS with the UCTE", CIGRE Session, C1-204, Paris, France, 2010.

[14] B. Marinescu, M. Badis and L. Rouco, "Large-scale power system dynamic equivalents based on standard and border synchrony", IEEE Trans. Power Syst., vol. 25, no. 4, pp. 1873-1882, 2010.

[15] B. Marinescu and L. Rouco, "A unified framework for nonlinear dynamic simulation and modal analysis for control of large-scale power systems", in Proc. IEEE 15th Power Sys. Comput. Conf., pp. 1-7, Liege, Belgium, 22-26 August, 2005.

[16] B. Meyer and M. Stubbe, "Eurostag, a single tool for power system simulation", in Proc. T\&D Int., Mars, 1992.

[17] I. Munteanu, B. Marinescu and F. Xavier, "Analysis of the interactions between close HVDC links inserted in an AC grid", in Proc. IEEE Power Ener. Soc. Ge., pp. 1-5, Chicago, Illinois, USA, July 16-20, 2017.

[18] A. Moharana, R. Varma and R. Seethapathy, "Modal analysis of induction generator based wind farm connected to series-compensated transmission line and line commutated converter high-voltage DC transmission line", Electr. Pow. Compo. Sys., vol. 42, no. 6, pp. 312-628, 2014.

[19] N. Martins, "The Dominant Pole Spectrum Eigensolver", IEEE Trans. Power Syst., vol. PWRS-12, no. 1, pp. 245-254, 1997.

[20] F. Mei and C. B. Pal, "Modal analysis of grid-connected doubly fed induction generators", IEEE Trans. Energy Convers., vol. 22, no. 3, pp. 728-736, 2007.

[21] I. J. Perez-Arriaga, G. C. Verghese and F. C. Schweppe, "Selective Modal Analysis with Applications to Electric Power Systems, PART I: Heuristic Introduction", IEEE Trans. Power Appar. Syst., vol. PAS-101, no. 9, pp. 3117-3125, 1982.

[22] R. Podmore, "Identification of Coherent Generators for Dynamic Equivalents", IEEE Trans. Power Appar. Syst., vol. PAS-97, pp. 1344-1354, 1978.

[23] G. N. Ramaswamy, G. C. Verghese, L. Rouco, C. Vialas and C. L. DeMarco, 'Synchrony, aggregation and multi-area eigenanalysis', IEEE Trans. Power Syst., vol. 10, no. 4, pp. 1986-1993, 1995.

[24] G. Rogers, Power system oscillations , Springer Sci. \& Bus. Media, Ed. 1, pp. XI-328, 2000.

[25] G. Rogers, Power system coherency and model reduction, New York: Springer, Ed. 1, vol. 94, pp. I-300, 2013.

[26] L. Rouco, I. J. Perez-Arriaga, R. Criado and J. Soto, "A computer package for analysis of small signal stability in large electric power systems", in Proc. IEEE 11th Power Sys. Comp. Conf., pp. 1141-1148, Avignon, France, 30 August, 1993.

[27] L. Rouco and I. J. Perez-Arriaga, "Multi-area analysis of small signal stability in large electric power systems by SMA", IEEE Trans. Power Syst., vol. PWRS-8, no. 3, pp. 1257-1265, 1993.

[28] R. Singh, M. Elizondo and S. Lu, "A review of dynamic generator reduction methods for transient stability studies", in Proc. IEEE Power. Ener. Soc. Ge., vol. 152, pp. 1-8, Detroit, MI, USA, 24-29 July, 2011.

[29] S. Skogestad and I. Postlethwaite, Multivariable feedback control: analysis and desig, New York: Wiley, vol. 2, pp. 359-368, 2007.

[30] M. Van De Wal and B. De Jager, "A review of methods for input/output selection", Automatica, vol. 37, no. 4, pp. 487-510, 2001.

[31] B. Wittenmark and M. E. Salgado, "Hankel-norm based interaction measure for input-output pairing", in Proc. IFAC 15th Triennial World Cong., pp. 429-434, Barcelona, Spain, 21-26 July, 2002.

[32] F. Xavier and B. Marinescu, "Analysis of a dynamic equivalent for representation of wind generation for modal analysis of large-scale power systems", in Proc. IEEE PowerTech, pp. 1-6, Grenoble, France, 16-20 June, 2013

[33] X. Yin and J. Liu, "Input-output pairing accounting for both structure and strength in coupling", AIChE J., vol. 63, no. 4, pp. 1226-1235, 2017. 


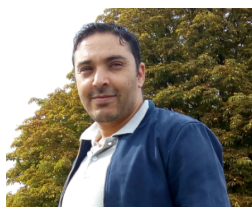

Kouki Mohamed was born in 1986 in Tunisia. $\mathrm{He}$ received the M.Sc degree in Electronic Engineering from the Faculty of Sciences of Tunis (FST) in 2011, and the Ph.D. degree in Electronic with straight honors from the Faculty of Sciences of Tunis (FST) in 2014. He is currently a Research Engineer in Ecole Centrale de Nantes in Laboratoire des Sciences du Numériques de Nantes (LS2N). His main resasch interests include model order reduction and modal-analysis of power systems.

Bogdan Marinescu was born in 1969 in Bucharest, Romania. He received the Engineering degree from the Polytechnical Institute of Bucharest in 1992, the $\mathrm{Ph} . \mathrm{D}$. degree from the Université Paris Sud-Orsay, France, in 1997, and the "Habilitation à diriger des recherches" from Ecole Normale Supérieure de Cachan, France, in 2010. He is currently a full Professor and Head of the chair "Analysis and control of power grids" in Ecole Centrale Nantes and head of the research team "Dynamics of Power Systems"in Laboratoire des Sciences du Numériques de Nantes. In the first part of his career, he was active in $R \& D$ divisions of industry (Électricité de France and Réseau de transport d'électricté) and as a parttime professor (especially from 2006 to 2012 in Ecole Normale Supérieure de Cachan). His main fields of interest are the theory and applications of linear systems, robust control and power systems engineering.

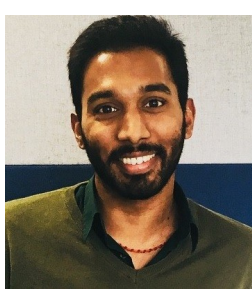

Florent Xavier Head of the Group "Integration of New Technologies" inside RTE's R\&D Direction, with 7 years of experience in the field of power systems. Graduated engineer from the École $\mathrm{Su}$ perieure d'Electricité; M.Sc in electrical and electronical engineering from the Swiss Federal Institute of Technology in Lausanne (EPFL). RTE representative in ENTSO-E groups: System Protection and Dynamic group (SPD), Research Development and Innovation Committee (RDIC) on System Stability working group and PG UA/MD. 